\title{
Seroprevalence of Trypanosoma cruzi in Rural Ecuador and Clustering of Seropositivity within Households
}

\author{
Carla L. Black, Sofía Ocaña-Mayorga, Diana K. Riner, Jaime A. Costales, Mauricio S. Lascano, \\ Laura Arcos-Terán, John S. Preisser, J. Richard Seed, and Mario J. Grijalva* \\ Department of Epidemiology, University of North Carolina, Chapel Hill, North Carolina; Center for Infectious Disease Research, \\ School of Biological Sciences, Pontifical Catholic University of Ecuador, Quito, Ecuador; Tropical Disease Institute, \\ Department of Biomedical Sciences, Ohio University College of Osteopathic Medicine, Athens, Ohio; \\ Department of Biostatistics, University of North Carolina, Chapel Hill, North Carolina
}

\begin{abstract}
We performed a cross-sectional study of Trypanosoma cruzi seroprevalence in 14 communities in three provinces of Ecuador and estimated the magnitude of the association of seropositive individuals within households. A total of 3,286 subjects from 997 households were included. Seroprevalence was $5.7 \%, 1.0 \%$, and $3.6 \%$ in subjects in the Manabí, Guayas, and Loja provinces, respectively. Seroprevalence increased with increasing age in Manabí and Guayas, whereas in Loja, the highest prevalence occurred in children $\leq 10$ years of age. In the coastal provinces, clustering of seropositives within households was not observed after adjustment for other household factors. However, in the Andean province of Loja, the odds of seropositivity were more than two times greater for an individual living in a household with another seropositive person. Our results indicate that transmission of T. cruzi is ongoing in Ecuador, although intensity of transmission and mechanisms of interaction between humans and the insect vectors of disease vary between geographic regions.
\end{abstract}

\section{INTRODUCTION}

The presence of human infection with Trypanosoma cruzi, the causative agent of Chagas disease, has been documented in Ecuador as early as 1927. During the 1940s and 1950s, new disease foci were reported from the coastal provinces of Guayas, Manabí, and Los Ríos and the Andean provinces of Loja, Azuay, and Bolívar. Today, Guayas and Manabí, along with the coastal province of El Oro, are thought to be the main endemic areas of the country. ${ }^{1}$ However, there are no recent reports of population-based studies assessing the current status of T. cruzi transmission in these areas. We report the prevalence of $T$. cruzi seropositivity in population-based samples of communities in rural areas of the Manabí, Guayas, and Loja provinces of Ecuador.

Another objective of this study was to estimate the extent to which $T$. cruzi infections cluster within households. In a paper published in 1976, Mott and others² stated "Since transmission of Chagas' disease largely occurs within households, description and analysis of the characteristics of household clustering of seropositivity to $T$. cruzi are of particular interest." Knowledge of the extent to which T. cruzi clusters within households can provide information about the extent to which an individual's risk of infection is affected by living in close proximity to another infected person who can serve as a reservoir of T. cruzi. Additionally, the persistence of clustering within households after adjustment for other known household risk factors for T. cruzi infection may indicate that other as yet unknown factors are involved in the transmission of T. cruzi within households. Mott and others ${ }^{2}$ reported significant clustering of T. cruzi seropositivity within households but were unable to quantify the degree of clustering or control for the effect of other household factors. ${ }^{2}$ A later analysis by Gurtler and others ${ }^{3}$ found no effect of clustering after

*Address correspondence to Mario J. Grijalva, Tropical Disease Institute, Biomedical Sciences Department, College of Osteopathic Medicine, Ohio University, 331 Irvine Hall, Athens, OH 45701. E-mail: grijalva@ohio.edu adjustment for other covariates using a random effects model. Although a random effects model can determine whether the parameter associated with clustering is statistically significant, the parameter itself does not have an easily understandable interpretation in terms of the magnitude of the clustering. We use the technique of alternating logistic regressions (ALRs) introduced by Carey and others ${ }^{4}$ to quantify the degree of clustering of T. cruzi infections within households. ALR provides pairwise odds ratios (PORs) of association of the outcome, in this case T. cruzi seropositivity, within clusters while also taking into account the dependence of the outcome on individual and cluster-specific covariates. The PORs obtained from ALR are interpreted similarly to conventional ORs, with a POR $>1$ indicating an association of seropositivity between individuals within a household. We also show how the POR can be used in the calculation of design effects, which is useful for planning the sample size of future studies in which subjects are sampled in households or other related units. ${ }^{4-6}$

\section{MATERIALS AND METHODS}

Study population. The subjects included in this study were residents of 14 rural communities in the Manabí, Guayas, and Loja provinces of Ecuador (Figure 1). Data were collected between June 2001 and August 2003. Manabí and Guayas are coastal provinces with a tropical climate, whereas Loja is located in the Andean highlands and has a temperate climate. Study personnel visited all households in the selected communities as part of a study of household risk factors for T. cruzi transmission. All members of each household were invited to attend study-sponsored medical clinics. All subjects that presented to the medical clinic and donated a blood sample to be tested for serologic evidence of T. cruzi infection were included in this study. Informed consent was obtained from all adult participants and parents of minor children. All study procedures were approved by the Institutional Review Boards of Ohio University and Catholic University of Ecuador. 


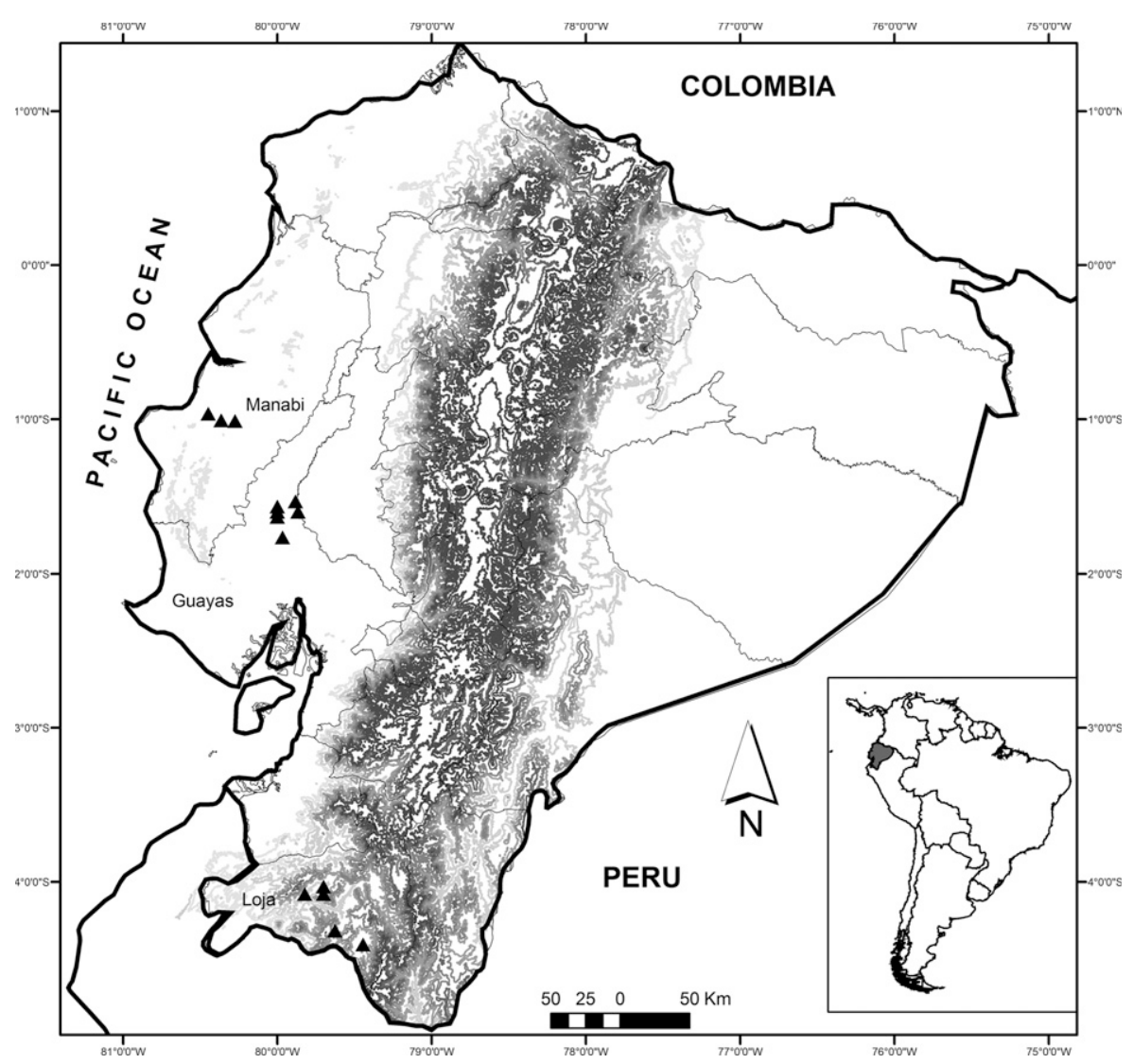

FIGURE 1. Map of Ecuador showing the elevation gradient as a gray scale, the provincial boundaries (solid lines), and the location of the studied communities (triangles).

A total of 4,530 subjects presented to the medical clinics, representing $80 \%$ of the eligible households in the study communities; 1,244 subjects were excluded from the analysis because they did not consent to serologic testing, leaving a final sample of 3,286 subjects.

Serologic testing. Blood was collected by venous puncture from all participants. A different serologic testing scheme was used in each of the three study sites. Initial screening in all three sites was performed by enzyme-linked immunosorbent assay (ELISA) using detergent-extracted T. cruzi epimastigote antigens as previously described. ${ }^{7}$ The optical density (OD) values of previously confirmed positive and negative controls were analyzed and used to define the limits for seropositivity and seronegativity of the assay. OD values within $2.5 \mathrm{SD}$ of the OD average for the positive controls were considered seropositive, and all $\mathrm{OD}$ values within $2.5 \mathrm{SD}$ of the $\mathrm{OD}$ average for negative controls were considered seronegative. Samples with OD values outside of the range of positive or negative were classified as borderline. Positive and borderline samples were assayed at least two more times. Samples that were positive at least three times were considered positive. Samples with repeated borderline results were considered negative.

For samples collected in the Manabí province, all samples positive by the initial ELISA were confirmed by immunofluorescence at the Centers for Disease Control and Prevention (Atlanta, GA), with a titer of 1:32 used as the positive cutoff. All samples collected in Guayas province, including those positive and negative by the initial ELISA, were also tested using a commercially available ELISA kit (Chagatest ELISA recombinante V 3.0; Wiener Laboratories, Rosario, Argentina) and a commercial indirect hemagglutination test (Chagatest HAI; Wiener Laboratories). All samples collected in the Loja province that were positive by the initial ELISA and $\sim 50 \%$ of the negative samples were subsequently tested with two commercial ELISA kits (Chagatest ELISA recombinante V 3.0; Wiener Laboratories, and Chagas Serum Antibody Detection Assay Microwell ELISA; IVD Research, Carlsbad, CA) and the Chagatest HAI (Wiener Laboratories). These tests were performed according to the manufacturer's instructions. Samples from Loja and Guayas that were positive by the recombinant ELISA or by at least two of the other tests were considered positive.

Analysis of prevalence. Poisson regression was used to estimate age-specific prevalences and corresponding 95\% confidence intervals (CIs) for each province. ${ }^{8,9}$ The log-linear regression model included a linear and a squared variable for age, two indicator variables corresponding to the three provinces, and terms for linear and squared age by province interactions. Age was coded as an ordinal variable ranging from 0 to 7 representing 10-year age categories. Variables for age squared and squared age by province interactions were included to improve model fit. The natural logarithm of the number of subjects in each 10-year age category in each province was included in the model as an offset term to provide a model for the rate of infections as opposed to the count. The fully specified model is shown in supplemental Appendix 1 (available online at www.ajtmh.org). 
The variance estimates used in the calculation of CIs were multiplied by the design effect to adjust for possible nonindependence of T. cruzi infections within households. The design effect is the amount by which the variance of the prevalence estimated under the assumption of simple random sampling must be inflated to account for the clustering of disease. The design effect (D) caused by the correlation of T. cruzi seropositivity within households was calculated from the PORs according to the following formula outlined by Katz and Zeger ${ }^{10}$ :

$$
\mathrm{D}=1+\left(\frac{p_{11}-p^{2}}{p(1-p)}\right)\left(\left[\frac{m-1}{N}\right] s^{2}+\bar{u}-1\right)
$$

where $p=$ prevalence of infection, $m=$ the number of households, $N=$ the total sample size, $\bar{u}=$ the mean of the household sizes, $s^{2}=$ the variance of the household sizes, and $p_{11}=$ the probability that two subjects chosen at random both have disease

$$
=\frac{1-2 p(1-\alpha)-\sqrt{[1-2 p(1-\alpha)]^{2}-4 a p^{2}(\alpha-1)}}{2(\alpha-1)}
$$

where $\alpha=$ within-household POR.

Estimation of the PORs is described below. The unadjusted PORs for each region were used in the calculation of the design effects.

Alternating logistic regressions. The magnitude of household clustering of $T$. cruzi seropositivity was estimated in the form of PORs of the association of seropositivity within households. The POR is interpreted as the increased odds in favor of seropositivity for an individual from a household where another individual chosen at random from that household is seropositive relative to the odds in favor of seropositivity if that randomly chosen individual is seronegative. ${ }^{5}$ PORs were estimated with the use of ALRs, as described by Carey and others. ${ }^{4}$ ALRs fit a model for the within-household OR while simultaneously adjusting for the effect of other covariates on the risk of seropositivity. A description of the ALR algorithm is given in supplemental Appendix 2 (available online at www .ajtmh.org).

Separate ALR models were constructed for the coastal provinces, which include Manabí and Guayas, and the Loja province, which is located in the Andean highlands. These two regions are geographically distinct with different insect vectors of T. cruzi, and a previous analysis of these data showed that household risk factors for $T$. cruzi differed between the two regions. ${ }^{11}$ In the previous analysis, type of materials used in the construction of roofs (palm or tile versus metal) and walls (cane or adobe versus cement) of houses were identified as risk factors for T. cruzi seropositivity. The presence of firewood and trash in the peridomicile area were additionally associated with seropositivity in the coastal provinces but not in the highlands. These factors were included as adjustment variables in the $\beta$ model of the ALRs. The resulting final models are shown in supplemental Appendix 2.

ALR analyses were performed with the GENMOD procedure in SAS version 8.2 (SAS, Cary, NC). An exchangeable structure was specified for the POR, assuming constancy across all households.
RESULTS

Prevalence of $\boldsymbol{T}$. cruzi seropositivity. The prevalence of T. cruzi seropositivity was 5.7\% (59/1041), 1.0\% (14/1343), and 3.6\% (32/902) in the studied communities of the Manabí, Guayas, and Loja provinces, respectively. Prevalence varied by community within each province (Table 1), ranging from $2.1 \%$ to $7.9 \%$ in Manabí, $0.6 \%$ to $2.0 \%$ in Guayas, and $1.2 \%$ to $7.2 \%$ in Loja.

Model-based age-specific prevalence estimates for each province are shown in Table 2 and Figure 2. In the Manabí province, prevalence increased with increasing age, ranging from $1.5 \%$ among children $<10$ years of age to a peak of $11.4 \%$ in persons $50-59$ years of age. Prevalence also increased with age in the Guayas province, from $0.3 \%$ among $0-9$ year olds to $2.4 \%$ in persons $\geq 70$ years of age. In the province of Loja, the highest prevalence of $7.1 \%$ occurred in children $<10$ years of age. Prevalence decreased with age until age 30, after which it remained steady at $\sim 2 \%$.

Clustering of $T$. cruzi seropositivity within households. In the coastal provinces of Manabí and Guayas, 73 seropositive subjects were identified from 693 households. Seven of these households had two cases living in the same house. The unadjusted POR for seropositivity within households was $1.42(95 \% \mathrm{CI}, 0.76,2.65)$. The design effect based on this estimate was 1.1. After adjustment for type of roof and the presence of firewood and trash in the peridomicile area, the POR was reduced to $0.97(0.54,1.74)$. A similar estimate of $0.96(0.52,1.77)$ was obtained in a model adjusted for wall type, firewood, and trash. Roof type and wall type were not included in the same model because of co-linearity between the two variables.

In the Loja province, 32 cases were identified in 304 households, with 5 households having 2 cases each. The unadjusted POR was $2.72(1.18,6.29)$, with a corresponding design effect of 1.8. Although housing construction materials were associated with $T$. cruzi seropositivity in the Loja province as well, these variables could not be included as covariates in the ALR model because no seropositive subjects lived in households

TABLE 1

Prevalence of $T$. cruzi seropositivity by community, Ecuador, 2001-2003

\begin{tabular}{lcc}
\hline Province and community & Number of cases/total sampled & Prevalence $(\%)$ \\
\hline Manabí & & \\
Cruz Alta & $42 / 534$ & 7.87 \\
Pasaje & $6 / 284$ & 2.11 \\
Pimpiguasí & $11 / 223$ & 4.93 \\
Total & $59 / 1,041$ & 5.67 \\
Guayas & & \\
La Alegría & $2 / 289$ & 0.69 \\
Lomas de Colimes & $2 / 220$ & 0.91 \\
Los Ángeles & $3 / 175$ & 1.71 \\
Macul & $2 / 337$ & 0.59 \\
Puerto Rico & $3 / 222$ & 1.35 \\
San Antonio & $2 / 100$ & 2.00 \\
Total & $14 / 1,343$ & 1.04 \\
Loja & & \\
Bramaderos & $6 / 173$ & 3.47 \\
Jacapo & $9 / 125$ & 7.20 \\
Naranjo Dulce & $9 / 179$ & 5.03 \\
Pindo Alto & $5 / 173$ & 2.89 \\
Playas & $3 / 252$ & 1.19 \\
Total & $32 / 902$ & 3.55 \\
\hline
\end{tabular}


TABLE 2

Estimated prevalence of T. cruzi seropositivity and $95 \%$ CIs by geographic region and age category, Ecuador, 2000-2002

\begin{tabular}{|c|c|c|}
\hline Region and age (years) & Number of cases/total sampled & $\begin{array}{c}\text { Estimated prevalence* }(\%) \\
(95 \% \mathrm{CI})\end{array}$ \\
\hline \multicolumn{3}{|l|}{ Manabí } \\
\hline $0-9$ & $3 / 184$ & $1.45(0.64,3.44)$ \\
\hline $10-19$ & $5 / 276$ & $2.96(1.82,4.81)$ \\
\hline $20-29$ & $13 / 159$ & $5.11(3.58,7.29)$ \\
\hline $30-39$ & $9 / 127$ & $7.67(5.32,11.07)$ \\
\hline $40-49$ & $10 / 103$ & $10.01(6.92,14.48)$ \\
\hline $50-59$ & $8 / 83$ & $11.35(7.96,16.17)$ \\
\hline $60-69$ & $6 / 58$ & $11.18(7.04,17.75)$ \\
\hline$\geq 70$ & $5 / 42$ & $9.56(4.32,21.15)$ \\
\hline \multicolumn{3}{|l|}{ Guayas } \\
\hline $0-9$ & $1 / 265$ & $0.33(0.07,1.68)$ \\
\hline 10-19 & $1 / 302$ & $0.54(0.20,1.45)$ \\
\hline $20-29$ & $1 / 214$ & $0.84(0.40,1.77)$ \\
\hline $30-39$ & $1 / 162$ & $1.20(0.56,2.58)$ \\
\hline $40-49$ & $3 / 149$ & $1.59(0.74,3.44)$ \\
\hline $50-59$ & $1 / 118$ & $1.96(0.96,4.01)$ \\
\hline $60-69$ & $3 / 69$ & $2.24(0.96,5.20)$ \\
\hline$\geq 70$ & $1 / 64$ & $2.37(0.59,9.59)$ \\
\hline \multicolumn{3}{|l|}{ Loja } \\
\hline $0-9$ & $7 / 99$ & $7.40(3.21,17.09)$ \\
\hline $10-19$ & $10 / 243$ & $4.20(2.42,7.29)$ \\
\hline $20-29$ & $4 / 111$ & $2.77(1.36,5.66)$ \\
\hline $30-39$ & 3/99 & $2.13(0.90,5.09)$ \\
\hline $40-49$ & 0/84 & $1.91(0.78,4.68)$ \\
\hline $50-59$ & 2/91 & $2.00(0.87,4.61)$ \\
\hline $60-69$ & $2 / 90$ & $2.44(0.98,6.04)$ \\
\hline$\geq 70$ & $3 / 77$ & $3.45(0.88,13.56)$ \\
\hline
\end{tabular}

constructed of cement walls or metal roofs, the referent categories for these variables. All seropositives lived in houses with adobe walls and tile roofs, with the exception of two cases living in a house without walls. An analysis restricted only to subjects from households with tile roofs and adobe or no walls reduced the within household association of T. cruzi seropositivity only slightly $(\mathrm{POR}=2.39 ; 1.05,5.44)$, indicating that factors other than shared exposure to substandard housing conditions are contributing to the clustering of infections within households. Although the presence of firewood and trash in the peridomicile was not identified as risk factors for seropositivity in the highlands region, a model containing these two variables was run to produce an estimate directly comparable to that from the coastal region. The POR result-

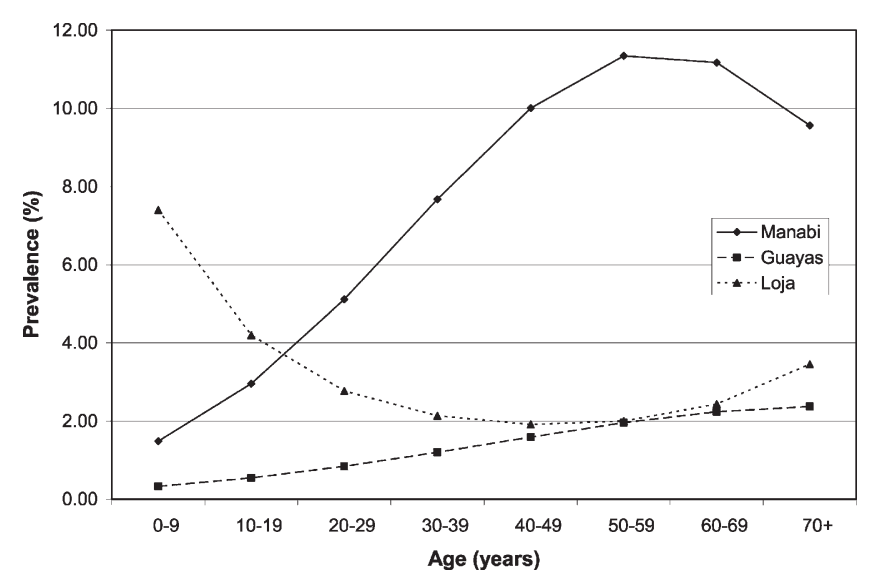

FIgURE 2. Prevalence of T. cruzi seropositivity by province and age category, Ecuador, 2001-2003. ing from this model was $2.51(1.08,5.81)$, also similar to the unadjusted estimate.

\section{DISCUSSION}

Our finding of $5.7 \%$ overall prevalence of $T$. cruzi seropositivity in the Manabí province is consistent with previous studies, which report seropositivity in various locations in Manabí ranging from $1 \%$ to $17 \% .{ }^{1}$ The pattern of agespecific prevalence is lowest in the youngest ages and steadily increases with increasing age, a pattern that is indicative of a chronic infection that has been endemic in the population for a long period of time. Many previous studies in areas of ongoing endemic $T$. cruzi transmission have shown a proportional increase in prevalence according to age. ${ }^{3,12-14}$ Our results indicate that $T$. cruzi infection remains a significant problem in this region, and there is no evidence of a reduction in transmission over the past 50 years. The continued transmission of T. cruzi is also supported by a recent entomologic survey from the same areas of Manabí, which found $21 \%$ of households to be infested with triatomine insects capable of serving as vectors of T. cruzi (MJ Grijalva, unpublished data).

Like Manabí, the province of Guayas is historically considered to be endemic for T. cruzi, with previous estimates of prevalence as high as $24 \%$ reported in $1959 .{ }^{1}$ We found an overall prevalence in Guayas of $1.0 \%$, an estimate lower than those from previous studies. A trend for increased prevalence with increasing age was observed, suggesting that T. cruzi was once endemic in this area. However, the prevalence was very low among those in the youngest age categories $(<1 \%$ in all age groups $<30)$. These results are indicative of a reduction or possible recent interruption of $T$. cruzi transmission in this area. An entomologic survey of 476 households in Guayas conducted in conjunction with this study found evidence of triatomine vectors in only one domicile (MJ Grijalva, unpublished data).

The prevalence of T. cruzi infection in the Loja province has not been widely studied. A prevalence rate of $2 \%$ from a 1955 survey of schoolchildren is the only previous report from this area that could be found in the medical literature. ${ }^{1}$ In this study, we found a prevalence of $7.1 \%$ among children $<10$ years of age, suggesting that active transmission of T. cruzi is occurring in this area. Prevalence did not increase with increasing age as would be expected if T. cruzi has been endemic in this area for many years. Conversely, prevalence actually decreased with age in the youngest age groups and remained steady after age 30 . This pattern suggests possible recent introduction or re-introduction of T. cruzi into this area. A similar result was seen in a recent study by Bowman and others, ${ }^{15}$ where prevalence rates were similar in adults and teenagers in an area of Peru where onset of transmission was estimated to have begun 11-12 years before the time of the survey. An entomologic survey conducted concurrently with this study in the same communities of Loja found a household infestation rate by triatomine insects of $35 \%$. The study also found evidence to suggest that the triatomine vectors in this area can colonize human dwellings in a relatively short period of time. ${ }^{16}$ This further supports the notion that humans have recently been exposed to triatomine vectors that previously circulated T. cruzi in wild ecotopes and have presently become domiciliated. 
Because different protocols for serologic testing were used in each province, the actual prevalence estimates are not directly comparable between provinces, although trends across age categories within provinces should be comparable. In the Manabí province, only those samples that were positive by the initial screening test were tested again, and only one additional test was performed on these samples. As such, this was the least sensitive and most specific testing scheme of the three sites, and the reported prevalence from Manabí likely underestimates the true prevalence. The testing scheme used in Guayas was the most sensitive and least specific, because every sample was tested with three separate tests. The true prevalence in the Guayas province was possibly even lower than what we reported.

Our prevalence estimates are also limited by a high nonparticipation rate. Although persons from $80 \%$ of the total households in the study communities reported to the clinics and were thus eligible for inclusion in the survey, we do not have data on the total number of people living in the nonparticipating households nor the number of the people in the participating households that did not report to the clinics. However, we do know that $75 \%$ of the 1,244 subjects that came to the clinics but were excluded from the analysis because of lack of serology were children $<10$ years of age. Had these subjects been included in the analysis, the resulting overall prevalence would likely be lower than the reported prevalence in Manabí and Guayas and higher than the reported prevalence in Loja.

In the coastal provinces, the degree of clustering of seropositives within households was small and not statistically significant. The value of the POR became very close to one after adjustment for shared household risk factors for T. cruzi infection, indicating that living in the same household as an infected person is not an independent risk factor for T. cruzi seropositivity. However, in the Loja province, the risk of seropositivity was more than two times greater if another randomly selected person from the same household was seropositive than if that randomly selected person was seronegative. The difference in household clustering might be explained by different behavior of the triatomine vectors in the two regions. As previously stated, household infestation with triatomine vectors of T. cruzi was found in both the Manabí and Loja provinces. In Loja, almost all captured insects were of the species Rhodnius ecuadoriensis (98\%), with small populations of Triatoma carrioni (2\%), Panstrongylus rufotuberculatus and Panstrongylus chinai (one bug each). ${ }^{16}$ In Manabí, the majority of captured insects were also R. ecuadoriensis (77\%), but a sizable population of Panstrongylus howardi (22\%) and a small number of P. rufotuberculatus ( $0.6 \%$ ) were also present (MJ Grijalva, unpublished data). However, even though the main vector of T. cruzi is the same in both regions, the entomologic situation is quite different. In Loja, bugs were almost exclusively intradomiciliary. Vectors were found in peridomicile areas only in occupied chicken nests. ${ }^{16}$ In Manabí, vectors were mainly peridomciliary. In $64 \%$ of infested houses, vectors were found solely in peridomicile areas such as piles of wood and brick and pens of domestic animals, with no bugs present in the intradomicile (MJ Grijalva, unpublished data). Evidence of colonization, indicated by the presence of triatomine eggs and or nymphs, was found in $85 \%$ of infested houses in Loja ${ }^{16}$ compared with $49 \%$ in Manabí (MJ Grijalva, unpublished data).
An analysis of risk factors for T. cruzi seropositivity conducted in this same population also supports the notion that differing behavior of triatomine vectors impacts where and how humans become infected. Although construction materials of houses were associated with seropositivity in both regions, peridomicile factors were not associated with seropositivity in Loja, which was expected as triatomine insects were found mainly inside houses. However, in the coastal region, the presence of firewood in the peridomicile increased the risk of seropositivity, possibly because vectors are passively transported into homes along with the firewood or because people are bitten as they carry the wood into their homes. Accumulation of trash and organic matter outside the home was protective against T. cruzi seropositivity. While a surprising finding, this could be an indication that vectors have colonized these areas rather than inside the domicile. ${ }^{11}$

In Manabí, where vectors are more likely peridomiciliary, if humans are being infected outside of the house or by vectors that only sporadically enter the house, an infected person within the house is not likely to serve as a reservoir of T. cruzi by which other triatomine insects can become infected. In Loja, where triatomines were found mostly in the intradomicile, humans are probably infected by vectors that have colonized their homes. Once infected, a person serves as a reservoir capable of infecting other insects inside the house, which in turn infect other persons sleeping in close proximity to the index case. Also, the percentage of seropositive individuals with detectable parasitemia has been reported to decline with age. ${ }^{17,18}$ Given the high prevalence of seropositivity among children in Loja, seropositive persons here likely have higher levels of parasitemia than seropositive persons in Manabí and Guayas, who are on average older and may have been infected for several decades. Thus, persons in Loja could be more infective to the triatomine vectors when bitten, and prevalence of $T$. cruzi infection in the vector population is more likely to be associated with human infections here than in the coastal regions, where rodents and domestic animals are probably more important sources of infection for the vectors. Another possible explanation for the observed clustering of seropositivity within households in Loja is that other unmeasured household characteristics are associated with T. cruzi transmission in this area.

Based on the PORs, we calculated design effects of 1.1 for the coastal region and 1.8 for Loja province. An estimation of design effects is often important when conducting populationbased surveys in developing countries where random sampling is not always feasible and subjects are routinely sampled by households, villages, or other related units. Although the design effect for the coastal region is small because of the low degree of household clustering in this region, a study of T. cruzi prevalence in Loja using a household sampling scheme would require a sample almost twice as large as a study design in which subjects were randomly sampled to produce prevalence estimates with equivalent precision. Because design effects are a function of within-cluster correlation, prevalence of disease, and cluster sizes, design effects can vary across studies. Relying on design effects reported from previous studies is not always useful for the planning of future studies. Because PORs are consistent across studies, PORs can be used to calculate design effects and estimate sample sizes for future studies when cluster sizes can be predetermined or predicted by the investigator and expected prevalence of the outcome is known. ${ }^{5,10}$ It should 
be noted that unadjusted PORs were used in the calculation of design effects. Even if household clustering of T. cruzi cases could be explained by shared household factors or other variables, the phenomenon that cases of T. cruzi are more likely to occur in the same household still exists and must be accounted for to produce accurate estimates of prevalence when a study design using household sampling is used.

In conclusion, we found evidence of T. cruzi infection in all three studied provinces of Ecuador. Prevalence patterns were suggestive of endemic infection in the Manabí province, reduction in transmission over time in the Guayas province, and recent introduction or re-introduction of transmission in the Loja province. T. cruzi infections clustered within households in the Loja province, whereas significant clustering was not observed in the coastal provinces. Differences in clustering between the regions might be caused by differing behavior of the triatomine vectors in each region or might be an indication that other unknown household factors play a role in the transmission of $T$. cruzi in the highlands region.

Received November 12, 2008. Accepted for publication June 25, 2009.

Note: Supplemental Appendices 1 and 2 can be found online at www ajtmh.org.

Financial support: This study received financial support from the UNICEF/UNDP/World Bank/WHO Special Programme for Research and Training in Tropical Diseases (TDR), PLAN Internacional Ecuador, Ohio University, and Catholic University of Ecuador.

Authors' addresses: Carla L. Black (current address), Center for Tropical and Emerging Global Diseases, University of Georgia, 500 D.W. Brooks Drive, Room 330, Athens, GA 30602, Tel: 706-542-4689, Fax: 706-542-3582, E-mail: blackc@uga.edu. Sofía Ocaña-Mayorga, Center for Infectious Disease, Research School of Biological Sciences, Pontifical Catholic University of Ecuador, Ed. Química Planta Baja \#1, Ave. 12 de Octubre y Roca, Quito, Ecuador, Tel: 593-2-2-991700 ext. 1856, Fax: 593-2-299-1687, E-mail: sbocana@puce.edu.ec. Diana K. Riner (current address), Emerging Infectious Diseases, Department of Microbiology and Immunology, Uniformed Services University of the Health Sciences, 4301 Jones Bridge Road, Bethesda, MD 20814, Tel: 301-295-3744, Fax: 301-295-1545, E-mail: diana.riner@usuhs.mil. Jaime A. Costales, Center for Infectious Disease, Research School of Biological Sciences, Pontifical Catholic University of Ecuador, Ed. Química Planta Baja \#1, Ave. 12 de Octubre y Roca, Quito, Ecuador, Tel: 593-2-2-991700, ext. 1856, Fax: 593-2-299-1687, E-mail: jacos talesc@puce.edu.ec. Mauricio S. Lascano, Tropical Disease Institute, Biomedical Sciences Department, College of Osteopathic Medicine, Ohio University, 331 Irvine Hall, Athens, OH 45701, Tel: 740-593 2192, Fax: 740-597-2778, E-mail: sl375903@ohio.edu. Laura Arcos-Terán, Center for Infectious Disease Research, School of Biological Sciences, Pontifical Catholic University of Ecuador, Ed. Ciencias 4to piso, Ave. 12 de Octubre y Roca, Quito, Ecuador, Tel: 593-2-2991576, Fax: 593-2299-1687, E-mail: 1arcos@puce.edu.ec. John S. Preisser, Department of Biostatistics, School of Public Health, University of North Carolina, 3105-F McGavran-Greenberg Hall, CB\# 7420, Chapel Hill, NC 27599, Tel: 919-966-7265, Fax: 919-966-3804, E-mail: jpreisse@bios.unc.edu. J. Richard Seed, Department of Epidemiology, School of Public Health, University of North Carolina, 2103-B McGavran-Greenberg Hall, CB\# 7435, Chapel Hill, NC 27599, Tel: 919-966-3527, Fax: 919-9662089, E-mail: rseed@email.unc.edu. Mario J. Grijalva, Tropical Disease Institute, Biomedical Sciences Department, College of Osteopathic Medicine, Ohio University, 331 Irvine Hall, Athens OH 45701, Tel: 740-593-2192, Fax: 740-597-2778, E-mail: grijalva@ohio.edu.

\section{REFERENCES}

1. Aguilar HM, Abad-Franch F, Racines J, Paucar A, 1999. Epidemiology of Chagas disease in Ecuador. A brief review. Mem Inst Oswaldo Cruz 94 (Suppl 1):387-393.

2. Mott KE, Lehman JS Jr, Hoff R, Morrow RD, Muniz TM, Sherlock I, Draper CC, Pugliese C, Guimaraes AC, 1976. The epidemiology and household distribution of seroreactivity to Trypanosoma cruzi in a rural community in northeast Brazil. Am J Trop Med Hyg 25: 552-562.

3. Gurtler RE, Chuit R, Cecere MC, Castanera MB, Cohen JE, Segura EL, 1998. Household prevalence of seropositivity for Trypanosoma cruzi in three rural villages in northwest Argentina: environmental, demographic, and entomologic associations. Am J Trop Med Hyg 59: 741-749.

4. Carey V, Zeger SL, Diggle P, 1993. Modeling multivariate binary data with alternating logistic regressions. Biometrika 80:517-526.

5. Katz J, Carey VJ, Zeger SL, Sommer A, 1993. Estimation of design effects and diarrhea clustering within households and villages. Am J Epidemiol 138: 994-1006.

6. Preisser JS, Arcury TA, Quandt SA, 2003. Detecting patterns of occupational illness clustering with alternating logistic regressions applied to longitudinal data. Am J Epidemiol 158: 495-501.

7. Grijalva MJ, Rowland EC, Powell MR, McCormick TS, Escalante $\mathrm{L}, 1995$. Blood donors in a vector-free zone of Ecuador potentially infected with Trypanosoma cruzi. Am J Trop Med Hyg 52: 360-363.

8. Koch GG, Atkinson SS, Stokes ME, 1986. Poisson regression. Kotz S, Johnson NL, eds. Encyclopedia of Statistical Sciences. Seventh edition. New York: John Wiley \& Sons, 32-41.

9. Stokes ME, Davis CS, Koch GG, 2000. Categorical Data Analysis Using the SAS System. Second edition. Cary, NC: SAS Institute.

10. Katz J, Zeger SL, 1994. Estimation of design effects in cluster surveys. Ann Epidemiol 4: 295-301.

11. Black CL, Ocaña S, Riner D, Costales JA, Lascano MS, Davila S, Arcos-Teran L, Seed JR, Grijalva MJ, 2007. Household risk factors for Trypanosoma cruzi seropositivity in two geographic regions of Ecuador. J Parasitol 93: 12-16.

12. Chippaux JP, Postigo JR, Santalla JA, Schneider D, Brutus L, 2008. Epidemiological evaluation of Chagas disease in a rural area of southern Bolivia. Trans R Soc Trop Med Hyg 102: 578-584.

13. Rizzo NR, Arana BA, Diaz A, Cordon-Rosales C, Klein RE, Powell MR, 2003. Seroprevalence of Trypanosoma cruzi infection among school-age children in an endemic area of Guatemala. Am J Trop Med Hyg 68: 678-682.

14. de Andrade ALSS, Zicker F, Luquetti AO, Oliveira RM, Silva SA, Souza JMP, Martelli CMT, 1992. Surveillance of Trypanosoma cruzi transmission by serological screening of schoolchildren. Bull World Health Organ 70: 625-629.

15. Bowman NM, Kawai V, Levy MZ, Cornejo del Carpio JG, Cabrera L, Delgado F, Malaga F, Benzaquen EC, Pinedo VV, Steurer F, Seitz AE, Gilman RH, Bern C, 2008. Chagas disease transmission in periurban communities of Arequipa, Peru. Clin Infect Dis 46: 1822-1828.

16. Grijalva MJ, Palomeque-Rodriguez FS, Costales JA, Davila S, Arcos-Teran L, 2005. High household infestation rates by synanthropic vectors of Chagas disease in southern Ecuador. J Med Entomol 42: 68-74.

17. Hoff R, Mott KE, Silva JF, Menezes V, Hoff JN, Barrett TV, Sherlock I, 1979. Prevalence of parasitemia and seroreactivity to Trypanosoma cruzi in a rural population of northeast Brazil. Am J Trop Med Hyg 28: 461-466.

18. Maguire JH, Mott KE, Hoff R, Guimaraes A, Franca JT, Souza JAA, Ramos NB, Sherlock IA, 1982. A three-year follow-up study of infection with Trypanosoma cruzi and electrocardiographic abnormalities in a rural community in northeast Brazil. Am J Trop Med Hyg 31: 42-47. 


\section{APPENDICES}

Appendix 1. Poisson regression model used to estimate age-specific prevalences for each

province.

The final model upon which estimates of prevalence $(\mathrm{Y} / \mathrm{N})$ are based is given as:

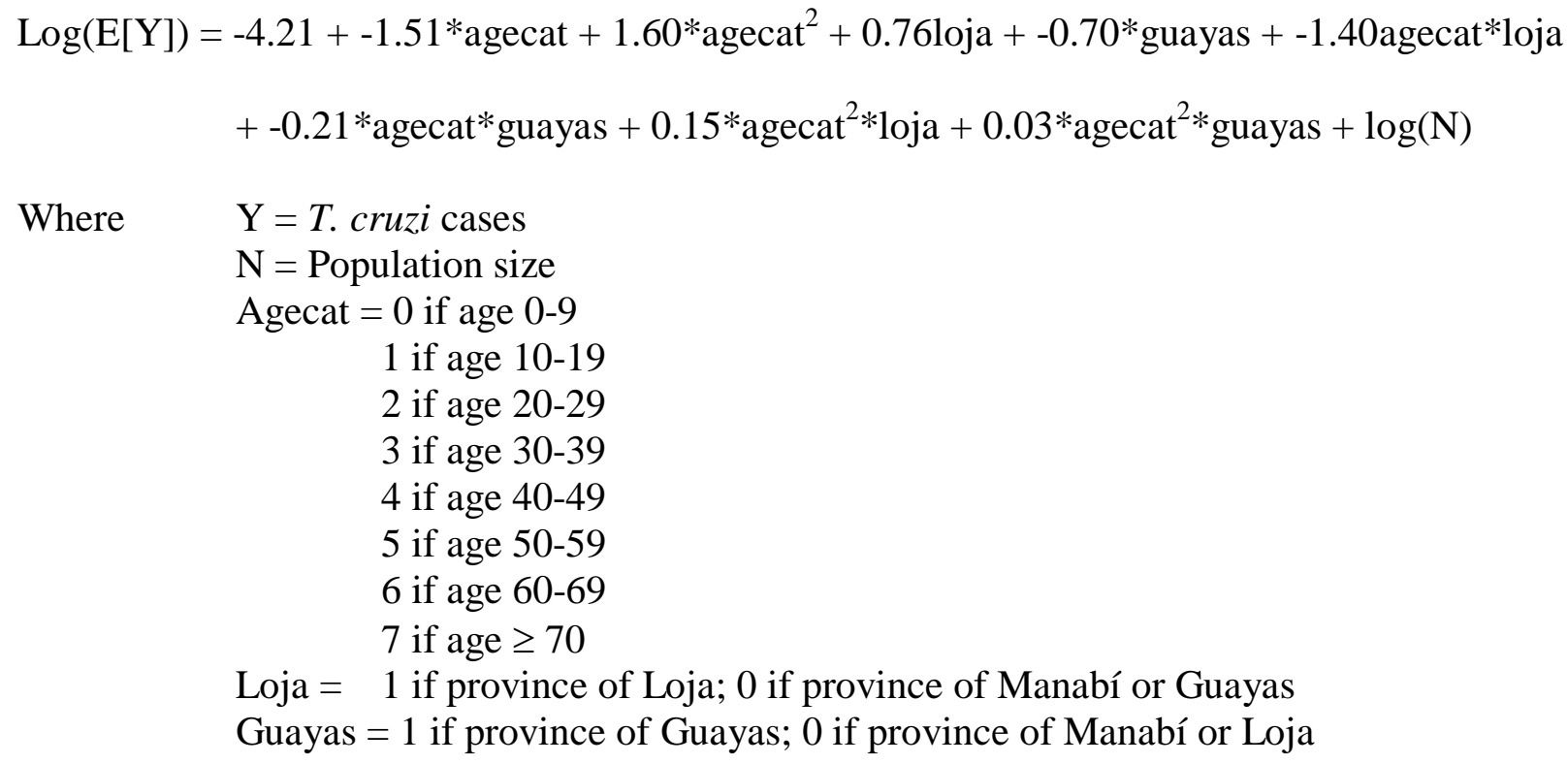




\section{Appendix 2. Estimation of pairwise odds ratios with the use of alternating logistic regressions}

The ALR algorithm involves the simultaneous estimation of two logistic regression models: one for the within-household pairwise odds ratios, given by $\log \left(\psi_{i j k}\right)=\alpha$, and one for the probability of seropositivity, given by logit $\operatorname{pr}(\mathrm{Y}=1)=\beta_{0}+\beta_{1} \mathrm{x}_{1}+\ldots+\beta_{\mathrm{p}} \mathrm{x}_{\mathrm{p}}$, where $\mathrm{x}_{1} \ldots \mathrm{x}_{\mathrm{p}}$ is a set of p explanatory variables associated with the risk of seropositivity and the $\beta$ s are the log odds ratios for the risk of seropositivity associated with the respective covariates. ${ }^{5}$ The pairwise OR between individual $j$ and individual $k$ within household $i$ is defined as

$\psi_{i j k}=\frac{\operatorname{pr}\left(Y_{i j}=1, Y_{i k}=1\right) \operatorname{pr}\left(Y_{i j}=0, Y_{i j}=0\right)}{\operatorname{pr}\left(Y_{i j}=1, Y_{i k}=0\right) \operatorname{pr}\left(Y_{i j}=0, Y_{i k}=1\right)}$

where $\mathrm{Y}=1$ if subject is seropositive; otherwise $\mathrm{Y}=0$ and $j \neq k^{4}$

Thus, the POR is interpreted as the increased odds in favor of seropositivity for an individual from a household where another individual chosen at random from that household is seropositive relative to the odds in favor of seropositivty if that randomly chosen individual is seronegative. ${ }^{5}$

An exchangeable structure for $\alpha$ was specified, which assumes that $j$ and $k$ are two randomly chosen individuals from the same household and the association between individuals is constant across all households.,

The logistic regression models used to obtain the PORs reported in this paper are as follows:

Coastal region, adjusted for roof type, firewood, and trash $\psi_{i j k}=-0.03$

logit $\operatorname{pr}(\mathrm{Y}=1)=-3.60+0.48 *$ roof $+0.66 *$ firewood $+-1.22 *$ trash 
Coastal region, adjusted for wall type, firewood, trash

$\psi_{i j k}=-0.04$

logit pr $(Y=1)=-4.11+1.39 *$ wallwood $+0.78 *$ wallcane $+0.67 *$ firewood $+-1.27 *$ trash

Loja province, unadjusted model

$\psi_{i j k}=1.00$

logit $\operatorname{pr}(\mathrm{Y}=1)=-3.30$

In each of the above models, $\mathrm{Y}=1$ if subject if seropositive, 0 otherwise

Roof $=1$ if house has palm roof, 0 otherwise

Wallwood = 1 if house has wood walls, 0 otherwise

Wallcane $=1$ if house has cane walls, 0 otherwise

Firewood $=1$ if presence of firewood in peridomicile, 0 otherwise

Trash $=1$ if presence of trash in peridomicile, 0 otherwise 